\title{
The rHEALPix Discrete Global Grid System: Considerations for Canada
}

\begin{tabular}{|r|l|}
\hline Journal: & Geomatica \\
\hline Manuscript ID & geomat-2018-0008.R1 \\
\hline Manuscript Type: & Research Article \\
\hline Date Submitted by the Author: & 21 -Jun-2018 \\
\hline $\begin{array}{r}\text { Complete List of Authors: } \\
\text { Is the invited manuscript for } \\
\text { consideration in a Special } \\
\text { Issue? : }\end{array}$ & $\begin{array}{l}\text { Sowater, David; University of New Brunswick, } \\
\text { Stefanakis, Emmanuel; University of New Brunswick }\end{array}$ \\
\hline $\begin{array}{r}\text { Keywords: } \\
\text { System }\end{array}$ & \begin{tabular}{l} 
Canada, rHEALPix DGGS, Spatial data structure, Discrete Global Grid \\
\hline
\end{tabular} \\
\hline
\end{tabular}

\section{SCHOLARONE \\ Manuscripts}




\title{
The rHEALPix Discrete Global Grid System: Considerations for Canada
}

\author{
David Bowater, Emmanuel Stefanakis \\ Department of Geodesy and Geomatics Engineering, University of New Brunswick, Canada
}

\begin{abstract}
Geospatial data is ubiquitous. However, the geographic grid is not designed to manage, store or integrate huge volumes of heterogeneous geospatial data. One possible solution to these challenges is a Discrete Global Grid System (DGGS) which was recently announced as a new standard by the Open Geospatial Consortium (OGC). If Canada plans to utilize a DGGS in the future to reference geospatial data, research is needed to explore characteristics and suitability of different DGGSs. Although hexagonal and triangular based DGGSs are popular, the square based rHEALPix DGGS mirrors the geographic grid more closely and has many advantages. This paper reviews key features of the rHEALPix DGGS, outlines recent work and qualitatively considers cell shape and cell orientation with respect to Canada, including how variations can be avoided or exploited by rotating the grid.
\end{abstract}

Keywords: Discrete Global Grid System; rHEALPix DGGS; Canada; Spatial data structure

\section{Introduction}

In recent years the growth in the number of ground, airborne, and satellite sensors has led to a significant increase in the amount, variety and rate of collection of geospatial data. However, combining huge volumes of heterogeneous geospatial data on the geographic grid is computationally expensive and time-consuming (OGC 2016; OGC 2017). One solution is a Discrete Global Grid System (DGGS). A DGGS is capable of efficient management, storage, integration, exploration, mining and visualisation of geospatial Big Data. Over the years many DGGSs have been devised and although a single DGGS cannot currently meet all global geospatial needs (Amiri et al. 2015; OGC 2017), the potential of a DGGS was confirmed in October 2017 when the Open Geospatial Consortium (OGC) officially adopted the DGGS as a new standard (OGC 2017c).

If Canada plans to adopt a DGGS framework in the future to reference geospatial data, important research is needed to explore characteristics and suitability of different DGGSs. While hexagonal and triangular DGGSs based on the ISEA (Icosahedral Snyder Equal Area) projection are popular, they are not optimal for all applications (Amiri et al. 2015). The rHEALPix DGGS (Gibb et al. 2016), which is based on the Hierarchical Equal Area isoLatitude Pixelization (HEALPix) projection, adopts a square cell approach that mirrors the geographic grid more closely and has several advantages over DGGSs based on hexagonal or triangular cells. This paper reviews key features of the rHEALPix DGGS, highlights advantages and disadvantages, outlines recent work and implementation, and qualitatively considers two 
fundamental characteristics with respect to Canada, namely cell shape and cell orientation, including how variations can be avoided or exploited by rotating the grid.

The content of this paper is organised as follows. Section 2 provides some background information relating to geographic grid issues, the Open Geospatial Consortium, and key terms in DGGS literature. Section 3 reviews the rHEALPix DGGS including recent work and implementation. Section 4 considers the rHEALPix DGGS with respect to Canada. Section 5 concludes the discussion by highlighting areas for future research.

\section{Background}

Discrete Global Grid System - four words that often bring blank or confused looks from inquiring minds in both academia and the public. A common definition says that a discrete global grid partitions the earth's surface into a series of cells, and a Discrete Global Grid System (DGGS) consists of many discrete global grids, that together provide a progressively finer resolution of cells (Sahr et al. 2003; Stefanakis 2016). If the meaning of a DGGS is still unclear, describing each word may help: Discrete refers to a finite number of cells, Global refers to a grid that completely covers the earth's surface, Grid refers to a tessellation of space into a uniform arrangement of cells, and System refers to a grid that can be subdivided into smaller cells. That being said, what is wrong with the traditional geographic grid and why is a DGGS preferred?

\subsection{Why use a DGGS?}

The geographic grid is a latitude-longitude parametrization of the sphere/ellipsoid (Amiri et al. 2015) originally designed for repeatable navigation and naturally suited to vector data, since the coordinate system references a continuous point field (Sahr et al. 2003; OGC 2017b). In order to create the quadrilateral cells that make up the geographic grid, equal angle steps are taken along the latitude and longitude axes.

While the geographic grid has many advantages, it contains inadequacies that make it impractical for many applications. For example, cell centroids are not equal distances apart, cells do not have uniform adjacency and cells surrounding the poles are actually triangles not quadrilaterals. These issues have been highlighted in several applications including climate modelling (Randall et al. 2002), numerical weather prediction (Carfora 2007) and oceanography (Hoggarth et al. n.d). Furthermore, grid cells do not have equal area (they gradually decrease in size from the equator to the pole) which complicates analyses such as sampling because cells do not have equal probabilities of being selected (White et al. 1992; OGC 2017b). Finally and perhaps most importantly, with the advent of Big Data, global data sets are now 
heterogeneous and high in volume. As a result, combining datasets from different sources (such as those having different sensors or coordinate reference systems) on the geographic grid is computationally expensive and time-consuming (OGC 2016).

On the other hand, a DGGS has many advantages that helps solve some of the problems faced by the geographic grid. Firstly, with regards to Big Data, a DGGS can easily manage, store and rapidly assemble large volumes of heterogeneous geospatial data which enables efficient exploration, mining and visualisation. Secondly, a DGGS can easily combine vector and raster data in the same framework thereby overcoming some of the difficulties faced by GIS approaches such as the 'vector-raster divide' and use of map projections (OGC 2017c). Finally, by adopting a grid of equal area cells, spatial analysis can be replicated regardless of location or resolution and information collected at a given location can be (i) referenced to the explicit area of its associated cell (ii) integrated with other cell values and (iii) provides statistically valid summaries from a sample of cells (OGC 2017b).

There have been many DGGSs devised since the 1980's and new interesting examples are still being created, such as the PlanetRisk DGGS (Sahr et al. 2015). A good summary of DGGSs up to 2003 can be found in (Sahr et al. 2003). Although there are a great variety of DGGSs, it should be noted that a single DGGS cannot currently provide the solution to all modern day geospatial applications. Having said that, they have been used separately for line simplification, optimal path determination, statistically valid sampling and indexing geospatial databases (Sahr et al. 2003). Furthermore, they can be optimized to meet many global geospatial needs including processing, analysis, visualization, and modelling (Stefanakis 2016; OGC 2017).

\subsection{The OGC}

In January 2016 the Open Geospatial Consortium (OGC) - an international organization that provides standards to the global community - issued a press release seeking comments on a DGGS standard that may replace those based on geographic coordinate systems (OGC 2016). In October 2017 the OGC officially adopted the DGGS as a new standard (OGC 2017c). This was an important announcement that could revolutionise the way geospatial information is used in the future. In addition, the OGC has set up a DGGS Standards Working Group (OGC 2017) with the aim of increasing awareness of DGGS advantages and to explore a standard that enables interoperability between Discrete Global Grid Systems. This has already prompted research on methods to aid index conversion between different DGGSs (Amiri et al. 2015) with more research likely to follow.

The new OGC DGGS standard entitled "Topic 21: Discrete Global Grid Systems Abstract Specification" includes important information on definitions, conventions, functional algorithms, the core 
data model and more (OGC 2017b). One section in particular, namely Global Grid Taxonomy, highlights the possible ways to create a global grid, only a few of which are classified as suitable under the abstract specification. Currently, three prominent options include the Snyder projection, rHEALPix projection and Quaternary Triangular Mesh (QTM). Notably, each is based on a different cell shape. Figure 1 shows examples of DGGSs that use these methods. Interestingly, PYXIS Innovation (PYXIS Innovation 2017) a Canadian company that has gained widespread attention for creating a new DGGS platform to analyse geospatial data - uses the ISEA3H (Icosahedral Snyder Equal Area Aperture 3 Hexagonal) DGGS (Sahr et al. 2003) whereas people at Landcare Research New Zealand created the rHEALPix DGGS (Gibb et al. 2016). This reiterates the fact that no single DGGS is optimal for all situations or preferred by all users.

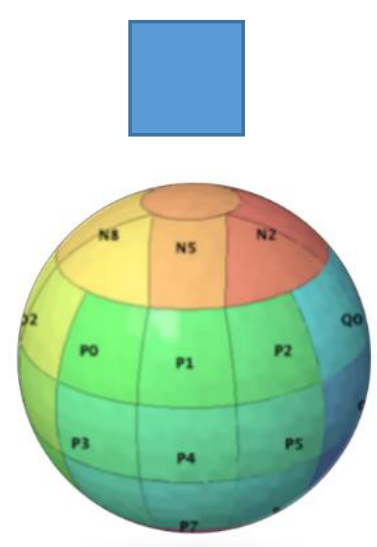

(a)

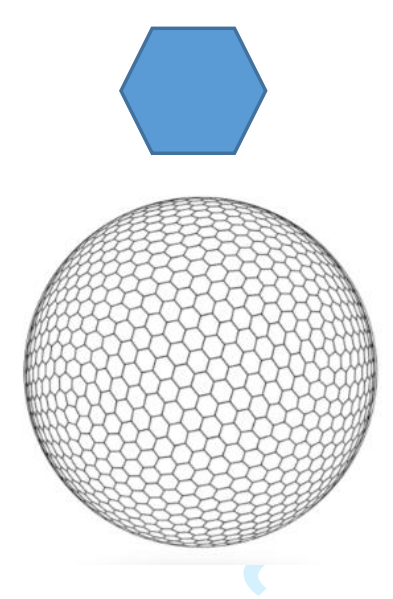

(b)

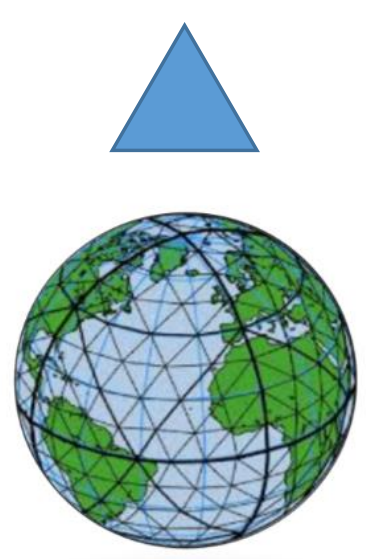

(c)

Figure 1. Examples of DGGSs and their corresponding cell shape. (a) Quadrilateral cells of the rHEALPix projection on the rHEALPix DGGS, (b) Hexagonal cells of the Snyder projection on the ISEA3H DGGS, and (c) Triangular cells of the QTM. (Copyright (c) 2017 Open Geospatial Consortium; OGC 2017b).

With regards to current software on the market for geospatial applications, PYXIS is leading the way. Its Digital Earth web based application makes it is easy to explore, combine, and analyse different data sets by adopting DGGS technology (PYXIS Innovation 2017). However, like any DGGS, the ISEA3H DGGS consists of specific design choices that mean it is not suited to every application. For example, it uses a hexagonal grid which is less familiar to users and less adaptable to Cartesian coordinate systems and hardware devices than traditional square grids (Amiri et al. 2015). In comparison to the hexagonal grid, quadrilateral grids mirror the geographic grid more closely. An emerging DGGS that adopts the quadrilateral grid approach is the rHEALPix DGGS. 


\subsection{Useful Terminology}

Before delving more into the rHEALPix DGGS, a few key terms should be clarified. First is base polyhedron. This refers to one of the five platonic solids that can tessellate a sphere/ellipsoid into equal area cells. See figure 2.

Second is refinement (sometimes called aperture). This refers to the process of subdividing a cell into a certain number of smaller cells. For example, if a planar square cell is subdivided into four squares cells of equal area, the refinement is called one-to-four, nine square cells is called one-to-nine and so on. See figure 3.

Third is resolution. This refers to the number of times the cell subdivision process has occurred. For example, assuming one-to-nine refinement, resolution one for a square has nine cells and resolution two has 81 cells. See figure 4.
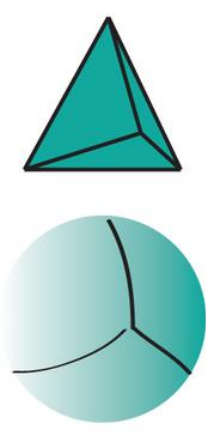

(a)
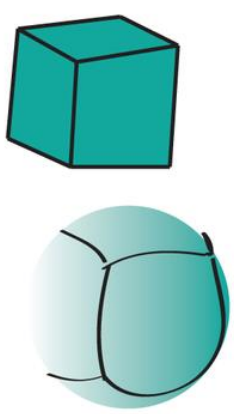

(b)

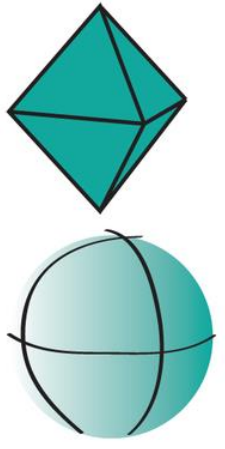

(c)

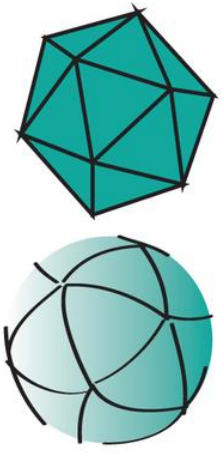

(d)

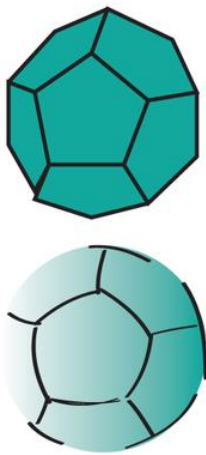

(e)

Figure 2. The five platonic solids and their spherical representation. (a) tetrahedron (b) hexahedron (cube) (c) octahedron (d) icosahedron (e) dodecahedron. (Copyright (c) 2017 Open Geospatial Consortium; OGC 2017b).

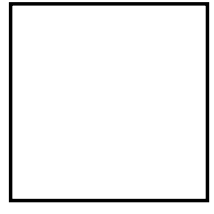

(a)

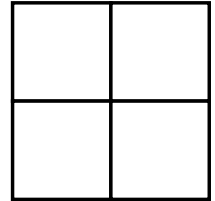

(b)

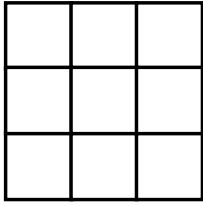

(c)

Figure 3. A square cell (a) with one-to-four refinement (b) and one-to-nine refinement (c). 


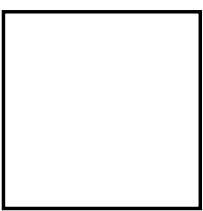

(a)

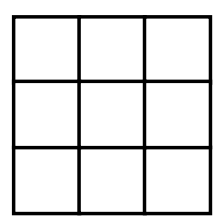

(b)

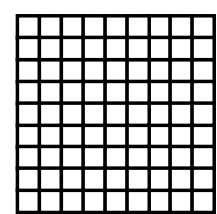

(c)

Figure 4. A square cell with one-to-nine refinement at resolution (a) 0 (b) 1 (c) 2.

With regards to cells, key terms include parent cell, child cell, aligned, congruent, and adjacency. A parent cell is a cell at resolution, $\mathrm{k}$, and a child cell is a cell at resolution, $\mathrm{k}+1$. Aligned means the centroid of the parent cell is also the centroid of a child cell. For example, this is true of a $3 \times 3$ (one-tonine refinement) square cell but not for a $2 \times 2$ (one-to-four refinement) square cell. See figure 5 .

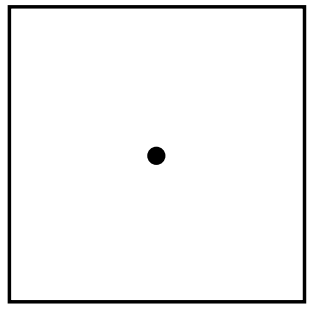

(a)

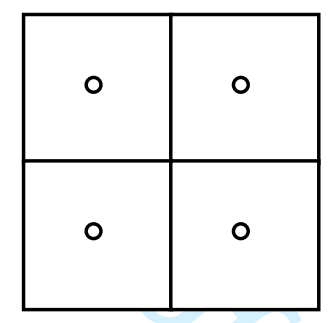

(b)

\begin{tabular}{|c|c|c|}
\hline$\circ$ & 0 & 0 \\
\hline 0 & $\bullet$ & 0 \\
\hline 0 & 0 & 0 \\
\hline
\end{tabular}

(c)

Figure 5. The centroid in the square parent cell (a) is not aligned with a child cell centroid in oneto-four refinement (b) but is aligned in one-to-nine refinement (c).

Congruent means the parent cell can be subdivided in such a way that child cells are enclosed by the parent cell. For example, squares and triangles are congruent but hexagons are not. See figure 6.

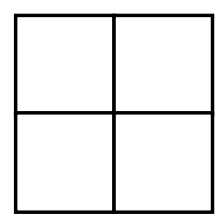

(a)

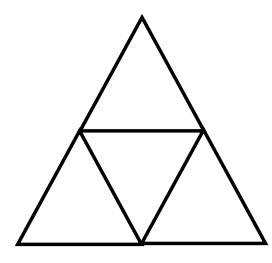

(b)

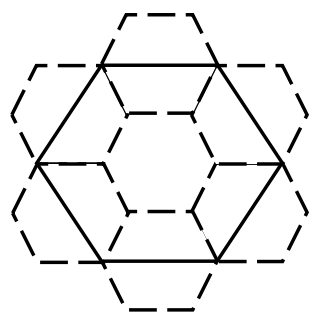

(c)

Figure 6. A square (a) and triangle (b) can be subdivided into child cells that are enclosed by the parent, whereas a hexagon (c) cannot be subdivided into hexagons that are enclosed by the parent. 
Adjacency refers to how a cell is connected to its neighbours. For example, hexagons exhibit uniform adjacency because they have six equal edge neighbours. Conversely, squares and triangles exhibit non-uniform adjacency because they have both edge and vertex neighbours (Sahr et al. 2003). See figure 7.

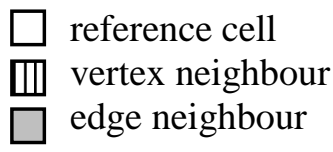
edge neighbour

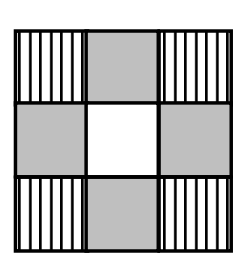

(a)

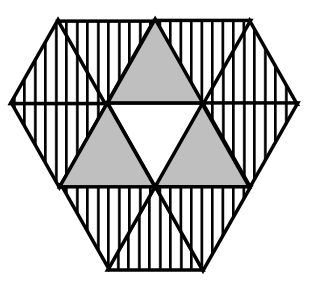

(b)

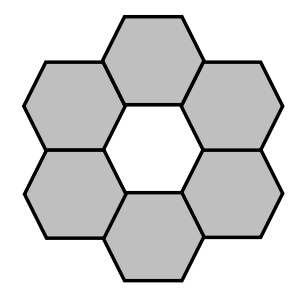

(c)

Figure 7. Edge and vertex neighbours of a square (a), triangle (b) and hexagon (c).

\section{The rHEALPix DGGS}

In general, DGGSs that have a planar square grid have many favourable characteristics including familiarity of square grids for users and compatibility with (i) existing hardware and display devices, (ii) quad-tree based algorithms, (iii) hierarchical data structures, and (iv) coordinate systems (Sahr et al. 2003; Gregory et al. 2008; Amiri et al. 2015). Although many innovative square based DGGSs have been designed, such as (Ma et al. 2009) and (Amiri et al. 2013), the rHEALPix DGGS appears most often in DGGS related articles and literature. In order to understand the rHEALPix DGGS, it is important to mention the HEALPix DGGS.

The HEALPix DGGS (Gorski et al. 2005) was developed out of necessity for use in the astronomical community. The motivation for HEALPix (Hierarchical Equal Area isoLatitude Pixelization) was linked to measurement and interpretation of cosmic background radiation. At the time, data sets covered the entire sky and were increasing at a rapid rate. As a result, a new DGGS was needed to process and analyse huge volumes of spherically distributed astronomical data in a timely and efficient manner. Consequently, a DGGS unique from any other alternative was created that had three essential features: (i) hierarchical tessellation, (ii) equal area cells at every resolution, and (iii) isolatitude distribution of cells (Isolatitude means cell nuclei are distributed only along lines of latitude). For example, the geographic grid does not satisfy (ii) and grids based on hexagonal and triangular tessellations do not satisfy (iii). Inquisitive readers can get more information about the background, purpose, history, as well as links to software at NASAs JPL website (JPL 2017). 
In 2016, people working at Landcare Research in New Zealand realized the potential of the HEALPix DGGS and extended it to be more suitable for geospatial needs, renaming it the rHEALPix DGGS (Gibb et al. 2016).

\subsection{Key Features}

In order for the rHEALPix DGGS to be more aligned with geospatial applications, the team made a few important amendments, including:

1. Compatibility with ellipsoids of revolution

2. Rearrangement of the planar projection into an alignment of square grids. See figure 8 .

3. Using a hexahedron as the base polyhedron.

Since reference ellipsoids (such as GRS80 and WGS84) are used in geodesy rather than spheres, the rHEALPix DGGS is more aligned with geospatial requirements and terrestrial use than its predecessor (Gibb 2016). Furthermore, by adopting a platonic solid as the base polyhedron, it is aligned with the criteria and design choices outlined in previous work on DGGS requirements for a digital earth framework (Sahr et al. 2003). Finally, by altering the planar projection to an alignment of square grids, it is easier to understand and link to similar grids used in remote sensing and environmental modelling (Gibb 2016). For detailed information on the rHEALPix DGGS including the definition, cell IDs, cell shapes, projection equations, distortions and mathematical underpinnings (see also (Calabretta et al. 2007)), readers are referred to (Gibb et al. 2016).

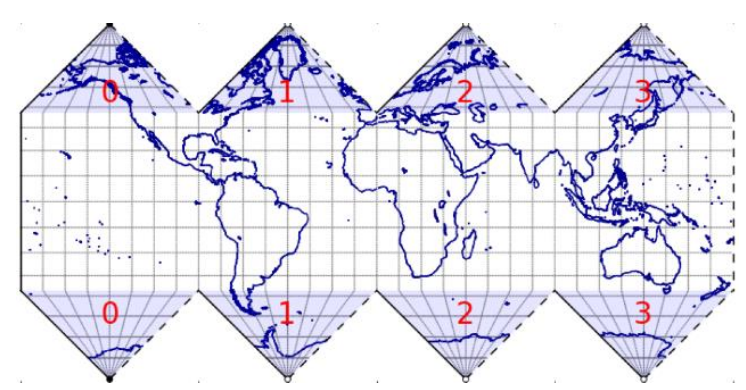

(a)

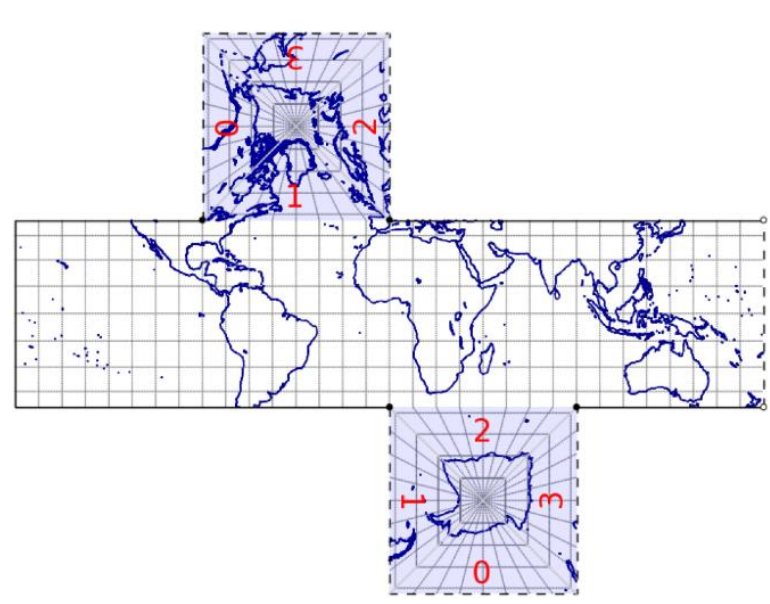

(b)

Figure 8. Combining the north and south polar triangles of the (a) HEALPix projection into two squares on the (b) rHEALPix projection. Grid lines represent parallels and meridians. (Gibb et al. 2016). 
The fundamental features of the rHEALPix DGGS that set it apart from other DGGSs include (Gibb 2016):

1. Hierarchical and congruent cell structure that is compatible with efficient data structures and easy to implement cell addressing schemes.

2. Aligned for odd factors of refinement, such as one-to-nine.

3. Equal area cells at every resolution.

4. Isolatitude distribution of cells which ensures fast computation of spherical harmonics.

5. Planar projection that has low average angular and linear distortion.

Two features that distinguish the rHEALPix DGGS from the popular the ISEA3H DGGS, are congruent cell structure, and isolatitude distribution of cells. Firstly, congruency is an important property because it makes hierarchical based cell indexing a trivial process which enables efficient handling of large volumes of data (Amiri et al. 2013; Amiri et al. 2015). Secondly, isolatititude distribution of cells is a rare quality in DGGSs. Since this feature is essential for fast computation of spherical harmonics, the rHEALPix DGGS is a good choice for applications that require harmonic analysis (Gibb et al. 2016). Therefore, geospatial applications involving tidal analysis or image processing for example, may find the rHEALPix DGGS well suited. Overall, the rHEALPix DGGS contains advantages both from its fundamental design and as a by-product of being a quadrilateral cell based grid. Furthermore, it contains features that other DGGSs simply do not have. As a result, the rHEALPix DGGS may be the ideal choice for new geospatial applications in the future.

It should be mentioned that the rHEALPix DGGS is not perfect for all applications and has limitations of use just like any other DGGS. The main disadvantages are lack of uniform adjacency and varying cell shape. The former, which is common in both quadrilateral and triangle cell based DGGSs, means distances to neighbouring cell centroids are not equal. This causes problems for dynamical systems where functions are related to intercell distances as well as in applications involving discrete simulations (Sahr et al. 2003; Gregory et al. 2008). The latter is a result of combining four triangular Collignon projections into a single square, see figure 8 . While all cells are quadrilaterals in the plane, some change shape when projected to the ellipsoid and four different cell shapes occur: the quad cell (quadrilateral), the dart cell (triangular), skew-quad cell (quadrilateral), and cap cell (circular) (Gibb et al. 2016). This is reminiscent of the geographic grid (triangular cells at the pole) and may lead to issues involving conversion algorithms between DGGSs or representation of geometries. Interestingly however, the rHEALPix DGGS can be rotated which means some cell shapes can be avoided in a given target area. 


\subsection{Recent Work and Implementation}

In 2016 the rHEALPix DGGS was updated in response to the proposed OGC core standard (OGC 2016b). The work included two main features. Firstly, it showed that cell geometries and cell IDs agree with the OGC standard. Secondly, it defined a process to determine cell adjacency and DE-9IM topological relationships using unique cell IDs rather than geodetic coordinates (Gibb 2016). This update was important work that promoted the usability and benefits of the rHEALPix DGGS, as well as its ability to be compliant with the proposed OGC core standard.

In addition, the rHEALPix DGGS has been implemented in Python and both the rHEALPix and HEALPix projections have been implemented in the Proj.4 Cartographic Library (Gibb et al. 2016). The Proj.4 library allows users to convert geodetic coordinates into and out of both projections using a command line window such as the OSGeo shell. Furthermore, it establishes the ground work for meeting the OGC requirement of having operations that allow data to be assigned to and retrieved from cells (Gibb 2016).

Research yielded one more example of implementation. A document prepared by the Spatial Data on the Web Working Group (a joint W3C [World Wide Web Consortium]-OGC project) described how earth observation data can be represented using the W3C RDF (Resource Description Framework) Data Cube and the rHEALPix DGGS. In particular, there is proof of concept that shows how satellite imagery can be retrieved from the Data Cube using a SPARQL query system (W3C 2017).

Currently, no further work or application utilising the rHEALPix DGGS can be found. With regards to modelling, visualisation and analysis of geographic entities, PYXIS have shown that DGGSs are a powerful tool. Could the rHEALPix DGGS provide new insights or applications in this field? As of yet, no research has been carried out. Future work should aim to determine how the well the rHEALPix DGGS is suited to handling geographic entities such as points, lines and polygons. Furthermore, due to the lack of open source algorithms that model geospatial entities on DGGSs, algorithms should be made available to the public to promote DGGS usability and reproducible science.

\section{Canadian Use}

The rHEALPix DGGS is classified as quadrilateral because its planar projection consists of a square grid. While the majority of planar squares are projected to quadrilateral cells, some are projected to circular or triangular cells. Although cell shapes vary, all cells have the same area regardless of shape so processes such as statistically valid sampling are not affected. In addition, cells shapes do not vary 
randomly on the ellipsoid, their positions can be calculated, accounted for, and somewhat avoided. In order to understand how this impacts Canada, the rHEALPix projection must be explained.

The rHEALPix DGGS uses the equal area rHEALPix projection to convert planar squares to ellipsoidal cells using the associated projection equations (Gibb et al. 2016). The rHEALPix projection is basically a rearrangement of the HEALPix projection, the mathematics for which can be found in (Calabretta et al. 2007). The details of the rearrangement and all projection equations can be found in (Gibb et al. 2016). This discussion will be based on the rHEALPix DGGS as described in (Gibb et al. 2016), in particular having a refinement of one-to-nine. The rHEALPix projection is comprised of two projections which form two regions: the Lambert cylindrical equal area projection in the equatorial region and the Collignon equal area projection in the polar region.

Somewhat unfortunately for Canada, the more user friendly and mathematically simpler equatorial region is defined by $\sim-41.9^{\circ} \leq \varphi \leq \sim 41.9^{\circ}$, where $\varphi$ is the geodetic latitude. This region adopts the Lambert cylindrical equal area projection which has the convenient property of projecting parallels to horizontal lines and meridians to vertical lines. As a result, a square in the plane becomes a quadrangle on the earth, known as a quad cell (Gibb et al. 2016) (a quadrangle is an area bounded by two parallels and two meridians). This means all cells in the equatorial region are quad cells. Figure 9 shows (a) quad cells on the ellipsoid and (b) Madagascar covered by a uniform quad cell grid.

\subsection{The Polar Region - Implications for Canada}

The polar region consists of the north polar region $\left(\varphi>\sim 41.9^{\circ}\right)$ and south polar region $(\varphi<$ $\sim-41.9^{\circ}$ ). Since they are isomorphic (Gibb et al. 2016) and Canada (almost entirely) lies in the north polar region, the discussion will be based there. Almost entirely, because Canada's most southerly location is in Ontario just below the equatorial/polar region border (e.g Pelee Island falls in the equatorial region). This is an important consideration if the area of interest is southern Ontario. Essentially though, all of Canada falls within the north polar region so equatorial region characteristics are not applicable.

The north polar region is created by rotating four triangular Collignon projections from the HEALPix projection into a single square. As a result, parallels project to straight lines (and appear as concentric squares) and meridians project to straight lines heading poleward, see figure $8 \mathrm{~b}$. This representation is quite difficult to understand, and visually determining projection coordinates from geodetic coordinates is not an easy task. While the mathematical formulas have been implemented in the Proj.4 library to carry out the conversion, this tool should be used with caution. Results can sometimes be unexpected and unfathomable without knowledge of the projection. Conversely, a square grid in the plane is projected to an ellipsoidal grid containing a variety of cell shapes. For refinement one-to-nine, there are 
three different cell shapes: the cap cell (circular), dart cell (triangular), and skew quad cell (quadrilateral), see figure 10. Note, if refinement is even then there are no cap cells (Gibb et al. 2016).

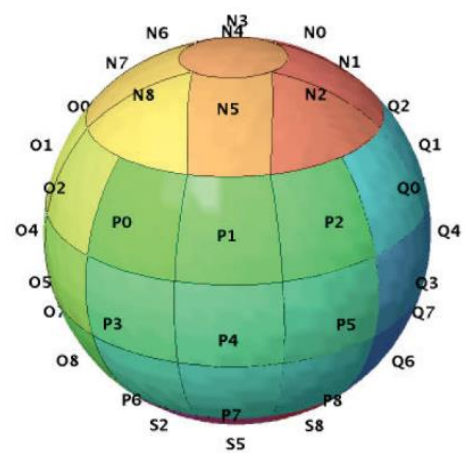

(a)

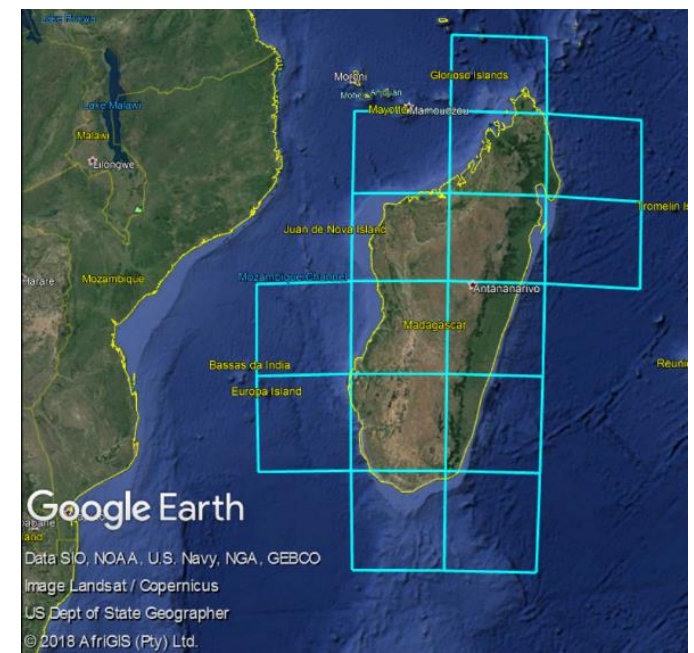

(b)

Figure 9. (a) Quad cells on the ellipsoid have letter prefixes O, P, Q and R (not visible) (Gibb 2016). (b) A grid of quad cells (at resolution 3) over Madagascar (latitude: $12^{\circ} \mathrm{S}-25.5^{\circ} \mathrm{S}$, longitude: $43^{\circ} \mathrm{E}-50.5^{\circ} \mathrm{E}$ approximately).

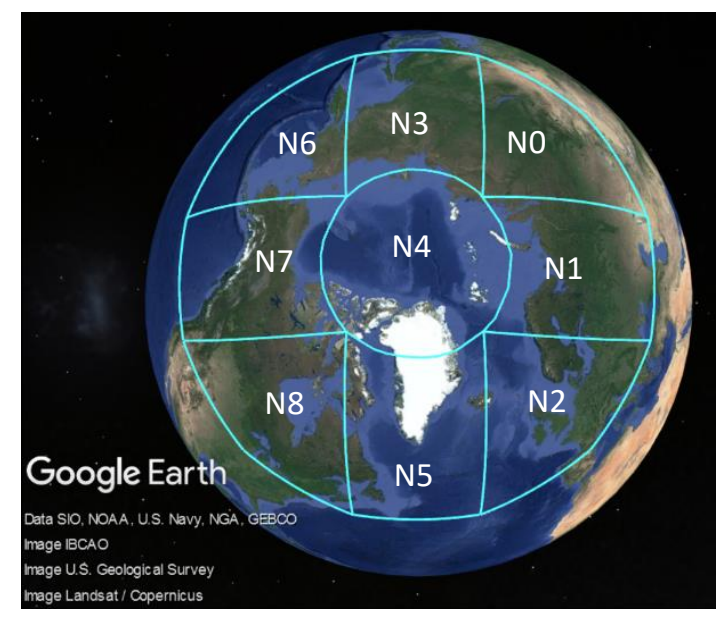

Figure 10. Cell shapes in the north polar region. N4 is the cap cell. N0, N2, N6 and N8 are dart cells. N1, N3, N5 and N7 are skew quad cells. Cell ID's correspond to figure 9a. 
The cap cell covers the pole and is the only circular cell. Its edge is a line of constant latitude with the cell centroid located at the pole. This cell is not a major concern for Canada because as resolution increases, the parallel defining the cell boundary converges towards the pole (since the cell area decreases and the centroid remains at the pole). Although Canada's northern boundary reaches high into the arctic, the likelihood of encountering the cap cell decreases with every increase in resolution, as shown in figure 11.

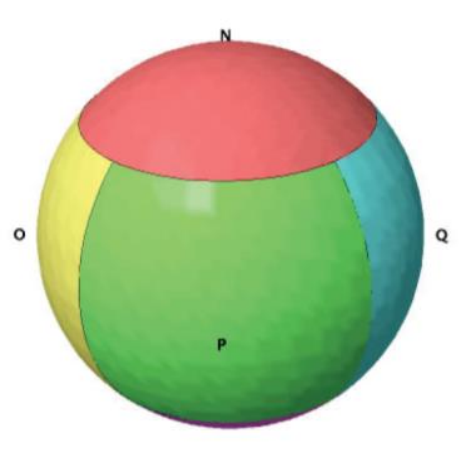

(a)

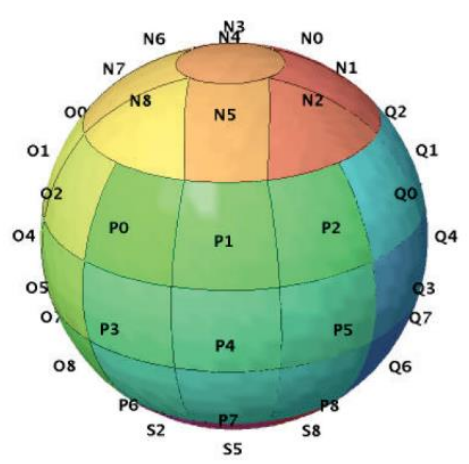

(b)

Figure 11. (a) The cap cell denoted $\mathrm{N}$ at resolution zero, and (b) the much smaller cap cell denoted N4 at resolution one. (Gibb 2016)

Triangular dart cells are formed where the edges of each of the four Collignon projections meet.

The southern edge of a dart cell is a line of constant latitude and north-east and north-west edges converge poleward to a point (Gibb et al. 2016). Furthermore, dart cells are aligned along four meridians and bounded by two parallels: the parallel that defines the cap cell and the parallel that defines the equatorial/ polar region border. This divides the north polar region into four quadrants spanning $90^{\circ}$. Since Canada has a large east-west extent that approaches $90^{\circ}$ in longitude, dart cells are unavoidable when considering the whole country (although they can be positioned at the east and west edges of Canada, see figure 12). However, if smaller features are of interest, such as the province of New Brunswick, dart cells can be avoided entirely.

The remaining cells in the north polar region are skew-quad cells. Skew-quad cells have four edges with the north and south edges aligned with parallels. In addition and only for skew quad cells, cell orientation changes with longitude through a quadrant, see figure 12. Consequently, cells initially having a north-west slant gradually rotate clockwise to a north-east slant. Similar to dart cells, this effect is unavoidable if considering the whole country but can be reduced over smaller areas. 


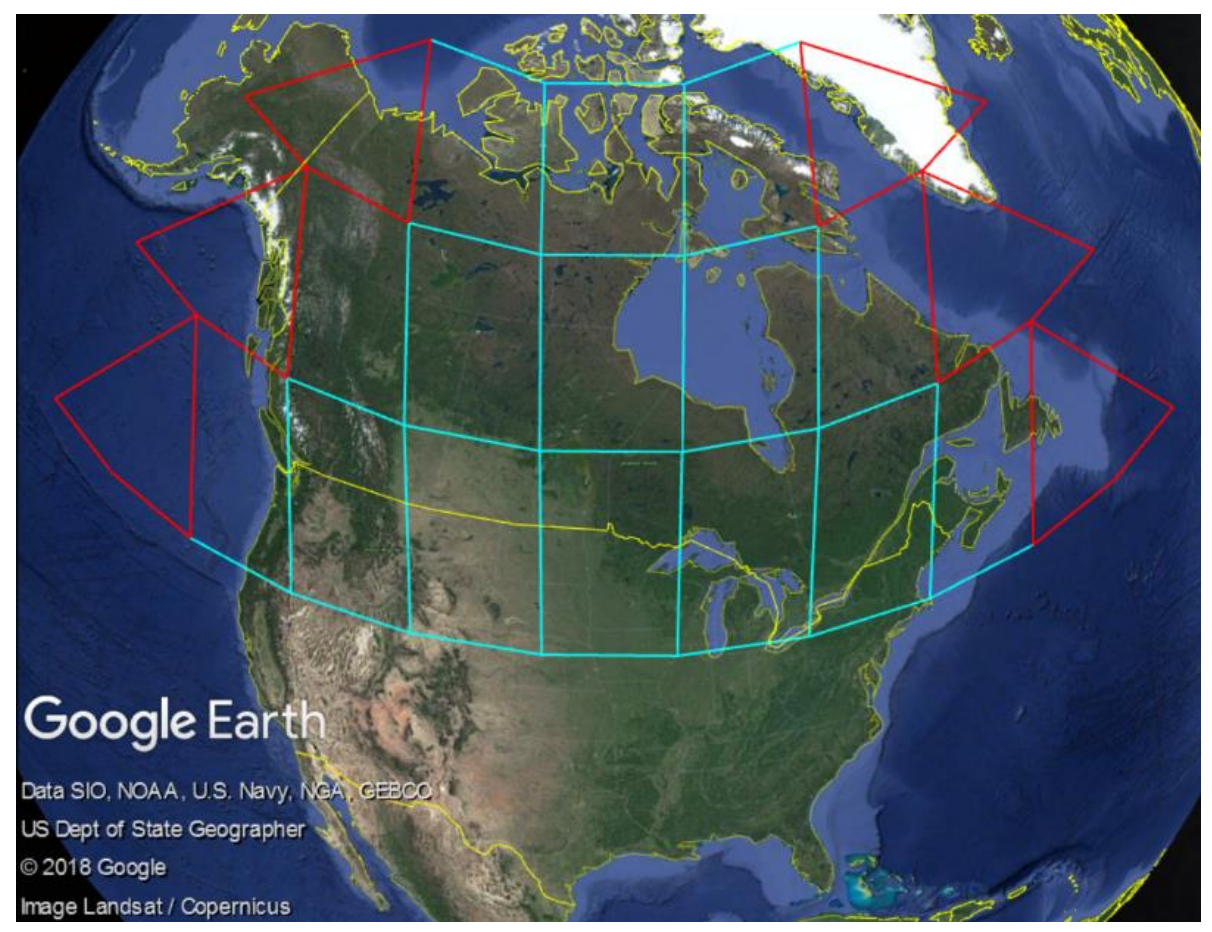

Figure 12. A grid (at resolution 2) of skew quad cells (cyan) and dart cells (red) over Canada. Dart cells can be positioned along the east and west edges of Canada. Orientation of skew quad cells is north-west in western Canada, north in central Canada and north-east in eastern Canada.

It has been established that a country the size of Canada in the polar region will have issues with varying cell shape and cell orientation. However, smaller areas can escape some of these issues, but how is this achieved? The answer lies in the versatility of the rHEALPix projection.

\subsection{Versatility of the rHEALPix projection}

An important advantage of the rHEALPix projection is its versatility, which means it can be rotated and altered to meet user needs. In order to tailor the rHEALPix projection to Canada, or any country in fact, it is possible to alter the rHEALPix projection in two ways. Firstly, the north polar square and south polar square can each be positioned in four different locations. Secondly, the projection image of the ellipsoid can be rotated by shifting the position of the prime meridian ( $0^{\circ}$ longitude), see (Gibb 2016).

By rearranging the north polar square, $n$, and south polar square, $s$, into four different locations, it is possible to create 16 different views of the projection. For example, figure 13 shows the $(n, s)=(0,0)$ and $(n, s)=(1,3)$ positions. Choosing a view is not a difficult task because the mathematics behind the projection enables the positions to be easily defined. The main advantage of this, is that it allows the projection to be rearranged to enable a better view of a chosen area (Gibb 2016). 
$\square$ equatorial square

$\square$ north polar square

III south polar square

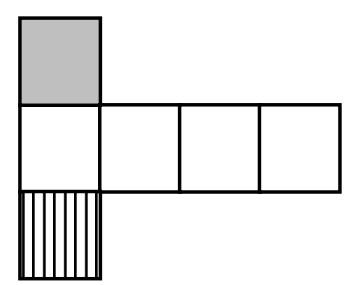

(a)

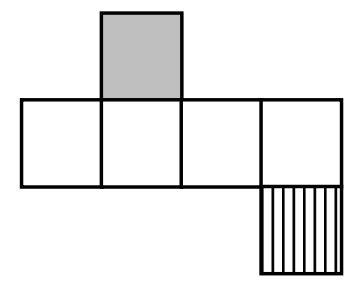

(b)

Figure 13. The rHEALPix projection in (a) $(n, s)=(0,0)$ position and (b) $(n, s)=(1,3)$ position.

The process of shifting the prime meridian simply involves adding the desired offset to all longitudinal values. This results in rotating the grid cells longitudinally around the ellipsoid. With regards to the polar region, this has two key benefits. Firstly, dart cells can be avoided because the four meridians on which they lie can be selected arbitrarily. While the four meridians will always have a $90^{\circ}$ separation, the positions of the meridians can be rotated to any location. Consequently, dart cells can be avoided for any area with a longitudinal extent less than approximately $90^{\circ}$. Since Canada has a longitudinal extent of approximately $90^{\circ}$, dart cells can almost be avoided by shifting the prime meridian to $50^{\circ} \mathrm{W}$. This aligns the dart cells with the $50^{\circ} \mathrm{W}$ and $140^{\circ} \mathrm{W}$ meridians, as shown in figure 14 .

Secondly, skew quad cells with angled orientations can be avoided or even exploited. This is an interesting feature because it provides some flexibility to choose cell orientation over a given area. For example, if an area is north-south aligned such as New Brunswick, skew quad cells having the same orientation can be used and a grid resembling the quad cell grid of the equatorial region to be achieved. Figure 15 shows a skew quad cell grid over New Brunswick at various resolutions where the prime meridian has been shifted to $21^{\circ} \mathrm{W}$. However, if an area has an angled orientation, such as Cape Breton, Nova Scotia, the grid can be rotated so that angled skew quad cells with similar orientation are used instead, see figure 16 where the prime meridian has been shifted to $50^{\circ} \mathrm{W}$. Notably, this characteristic only applies for a specific range of orientations. The most angled skew quad cells are adjacent to dart cells and have orientations of approximately $\mathrm{N} 30^{\circ} \mathrm{E}$ and $\mathrm{N} 30^{\circ} \mathrm{W}$. Therefore, using angled skew quad cells is most suited to areas having an orientation in this $60^{\circ}$ range.

With regards to the equatorial region, shifting the prime meridian rotates the grid so that quad cells can be more aligned with a given area. However, since quad cells are always aligned with parallels and meridians, shifting the prime meridian does not affect cell orientation. 


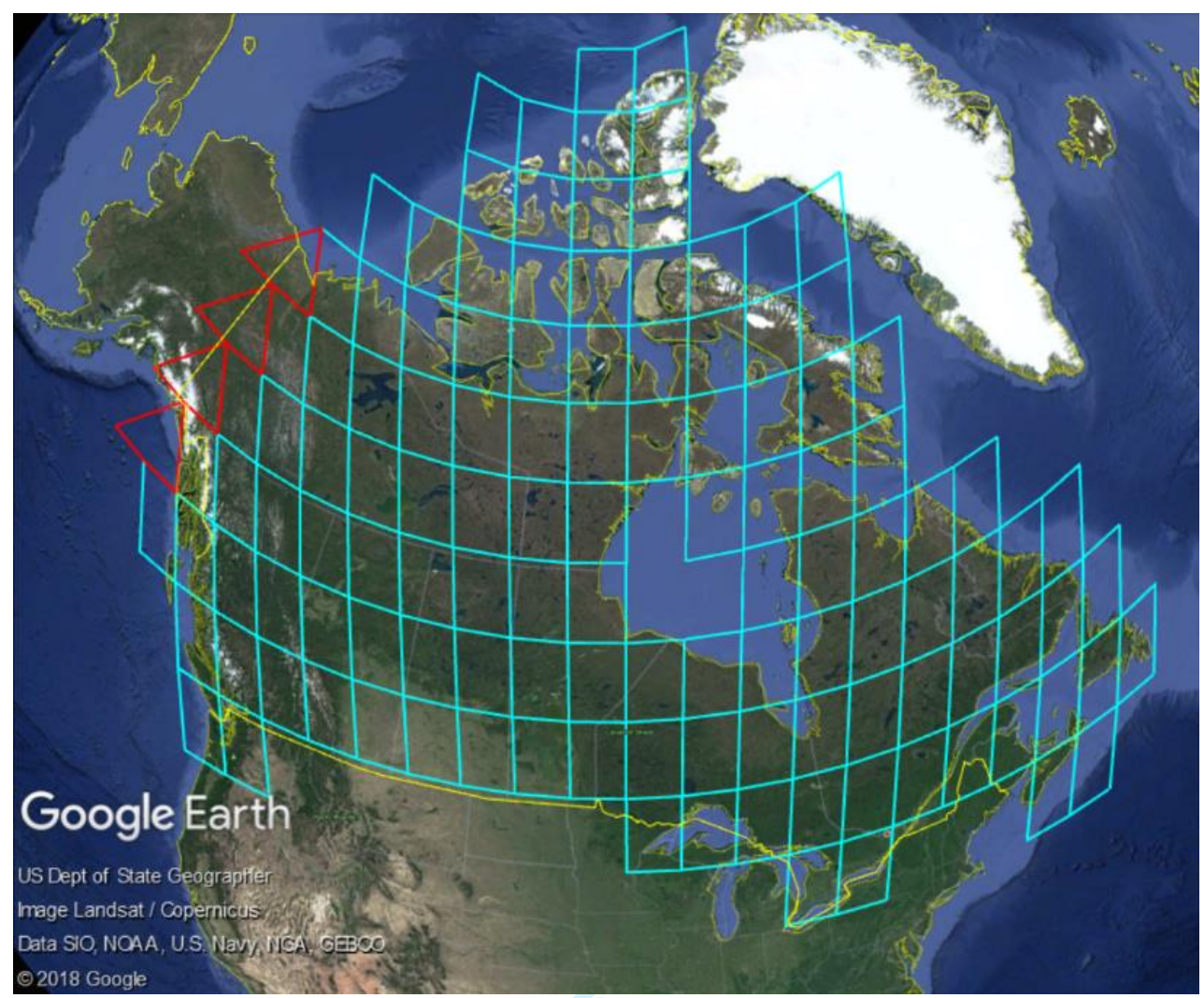

Figure 14. A rotated grid (at resolution 3) over Canada consisting of skew quad cells (cyan). Dart cells (red) can be seen along the Alaskan border. The prime meridian has been shifted to $50^{\circ} \mathrm{W}$.

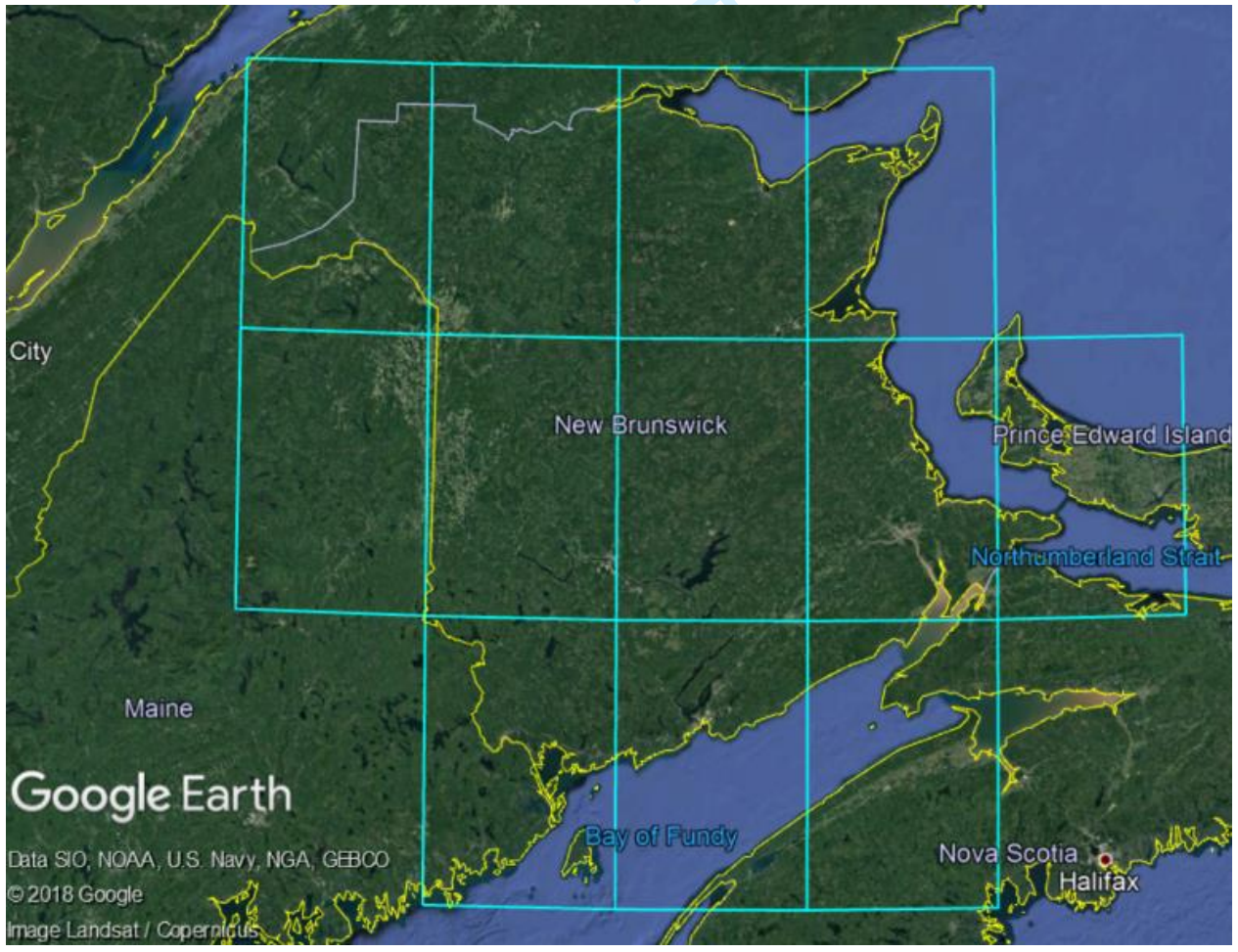

(a) 


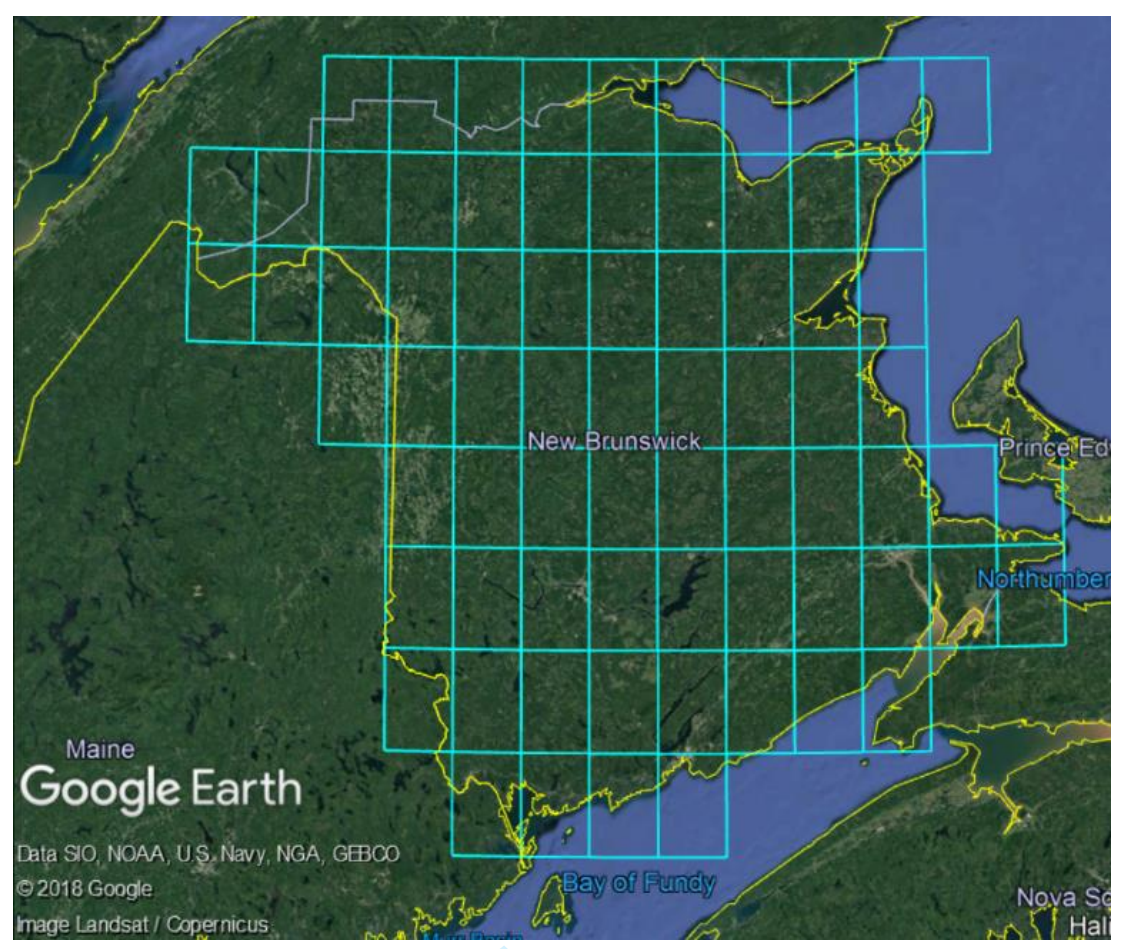

(b)

Figure 15. A grid of skew quad cells orientated to suit New Brunswick at resolution (a) 4 (b) 5. The prime meridian has been shifted to $21^{\circ} \mathrm{W}$.

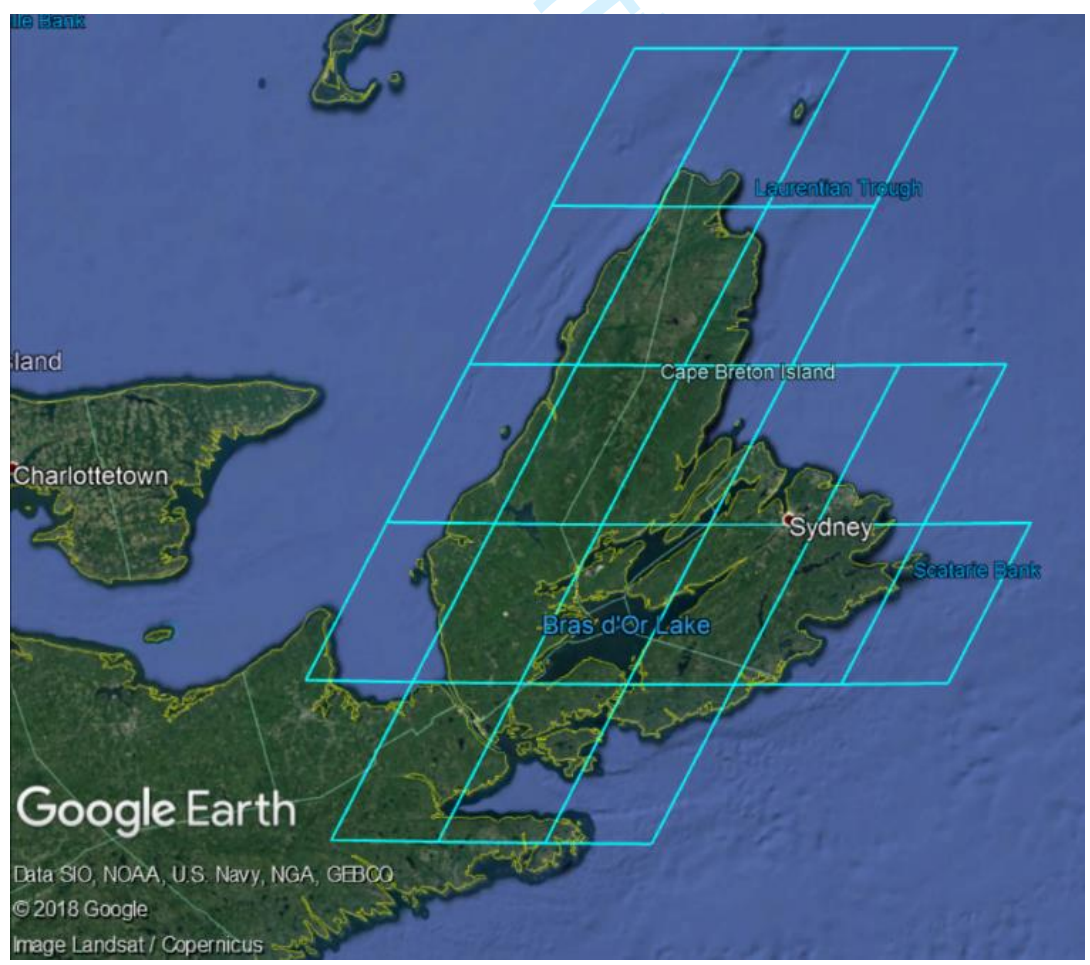

Figure 16. A grid of skew quad cells orientated to suit Cape Breton at resolution 5. The prime meridian has been shifted to $50^{\circ} \mathrm{W}$. 


\section{Conclusion}

This paper reviews key features of the rHEALPix DGGS, highlights recent work and implementation, and gives qualitative consideration to the rHEALPix DGGS over Canada. Although the rHEALPix DGGS is well defined, no significant research has been carried out to determine its ability to handle geospatial entities. For Canada, varying cell shape and cell orientation are key considerations due to the size and location of the country. However, for smaller features such as provinces, these variations can be avoided or even exploited by rotating the grid.

Future work should explore how geospatial entities such as points, lines and polygons can be modelled, visualized, and analysed on the rHEALPix DGGS. This will enable quantitative comparisons to be made against other DGGSs and help to determine the most suitable DGGS for Canada. Finally, due to the lack of open source algorithms that model geospatial entities on DGGSs, algorithms should be made available to the public to promote DGGS usability and reproducible science.

\section{Acknowledgements}

The authors wish to thank the editor and anonymous reviewers for their valuable comments. This work was funded by the Natural Sciences and Engineering Research Council of Canada (NSERC-DG).

\section{References}

Amiri, A. M., Bhojani, F., and Samavati, F., 2013. One-to-Two-Digital Earth. Proceedings of the International Symposium of Visual Computing, Springer, LNCS 8034, pp. 681-692.

Amiri, A. M., Samavati, F., and Peterson, P., 2015. Categorization and Conversions for Indexing Methods of Discrete Global Grid Systems. ISPRS International Journal of Geo-Information. 4, 320-336.

Calabretta, M. R., and Roukema, B. F., 2007. Mapping on the HEALPix grid. Monthly Notices of the Royal Astronomical Society. 381, 865-872.

Carfora, 2007. Interpolation on spherical geodesic grids: A comparative study. Journal of Computational and Applied Mathematics. 210(1-2), 99-105.

Gibb, R. G., 2016. The rHEALPix Discrete Global Grid System. Institute of Physics Conference Series: Earth and Environmental Sciences. 34, 012012.

Gibb, R., Raichev, A., and Speth, M., 2016. The rHEALPix Discrete Global Grid System. doi: 10.7931/J2D21VHM. Available online https://datastore.landcareresearch.co.nz/dataset/rhealpix-discreteglobal-grid-system (accessed on 16 May 2017). 
Gorski, K. M., Hivon, E., Banday, A. J., Wandelt, B. D., Hansen, F. K., Reinecke, M., and Bartelmann, M., 2005. HEALPix: a framework for high-resolution discretization and fast analysis of data distributed on the sphere. The Astrophysical Journal. 622, 759-771.

Gregory, M. J., Kimerling, A. J., White, D., and Sahr, K., 2008. A comparison of intercell metrics on discrete global grid systems. Computers, Environment and Urban Systems. 32(3), 188-203.

Hoggarth, A., and Taylor, T., n.d. The OGC Discrete Global Grid Systems core standard and its relevance to the hydrographic community. Available online https://www.iho.int/mtg_docs/com_wg/IRCC/IRCC8/IRCC8-07E-MSDIWG-AnnexH-GGS-forHydrography.pdf (accessed on 12 October 2017).

JPL, 2017. Healpix. http://healpix.jpl.nasa.gov/ [Visited on 6 October 2017].

Ma, T., Zhou, C., Xie, Y., Qin, B., and Ou, Y., 2009. A discrete square global grid system based on the parallels plane projection. International Journal of Geographical Information Science. 23(10), 1297 1313.

OGC, 2016. OGC seeks public comment on a candidate standard that may replace legacy coordinate systems. http://www.opengeospatial.org/pressroom/pressreleases/2349/ [Visited on 29 May 2017].

OGC, 2016b. OGC Discrete Global Grid System (DGGS) Core Standard. Available online http://www.opengeospatial.org/standards/requests/143 (accessed on 19 September 2017).

OGC, 2017. Discrete Global Grid Systems SWG. http://www.opengeospatial.org/projects/groups/dggsswg/ [Visited on 29 May 2017].

OGC, 2017b. Topic 21: Discrete Global Grid Systems Abstract Specification. Available online http://docs.opengeospatial.org/as/15-104r5/15-104r5.html (accessed on 3 January 2018).

OGC, 2017c. OGC announces a new standard that improves the way information is referenced to the earth. http://www.opengeospatial.org/pressroom/pressreleases/2656 [Visited on 3 January 2018].

PYXIS Innovation, 2017. https://www.pyxisinnovation.com/ [Visited on 6 October 2017].

Randall, D. A., Ringler, T. D., Heikes, R. P., Jones, P., and Baumgardner, J., 2002. Climate Modeling with Spherical Geodesic Grids. Computing in Science \& Engineering. 4(5), 32-41.

Sahr, K., White, D., and Kimerling, A. J., 2003. Geodesic discrete global grid systems. Cartography and Geographic Information Science. 30(2), 121-134. 
Sahr, K., Dumas, M., and Choudhuri, N., 2015. The PlanetRisk Discrete Global Grid System. Available online http://www.discreteglobalgrids.org/wp-content/uploads/sites/33/2016/10/PlanetRiskDGGS.pdf (accessed on 5 January 2018).

Stefanakis, E., 2016. Discrete Global Grid Systems - A new OGC standard emerges. GoGeomatics. Magazine of GoGeomatics Canada. January 2016.

White, D., Kimerling, J. A., and Overton, S. W., 1992. Cartographic and Geometric Components of a Global Sampling Design for Environmental Modelling. Available online http://capblanc.org/attachments/White_etal_CAGIS_1992.pdf (accessed on 10 January 2018).

W3C, 2017. Publishing and Using Earth Observation Data with the RDF Data Cube and the Discrete Global Grid System. https://w3c.github.io/sdw/eo-qb/\#DGGS [Visited on 6 October 2017]. 